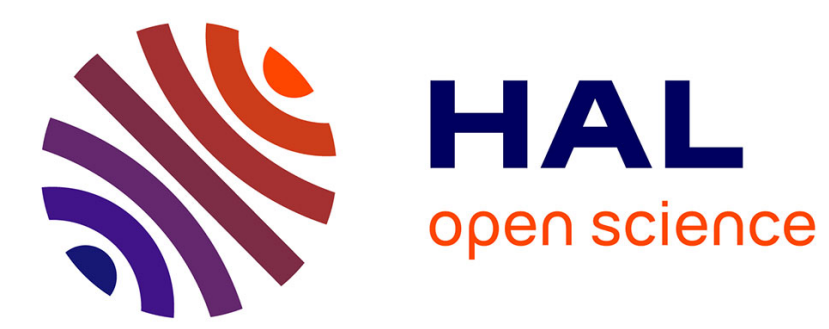

\title{
Multi-Object association with belief functions: the dual decision algorithm
}

\author{
Jean-Philippe Lauffenburger, Jérémie Daniel, Osamah Saif
}

\section{To cite this version:}

Jean-Philippe Lauffenburger, Jérémie Daniel, Osamah Saif. Multi-Object association with belief functions: the dual decision algorithm. Advances in Control and Automation Theory for Transportation Applications, Sep 2013, Istanbul, Turkey. pp.TuDT2. hal-00868778

\section{HAL Id: hal-00868778 https://hal.science/hal-00868778}

Submitted on 1 Oct 2013

HAL is a multi-disciplinary open access archive for the deposit and dissemination of scientific research documents, whether they are published or not. The documents may come from teaching and research institutions in France or abroad, or from public or private research centers.
L'archive ouverte pluridisciplinaire HAL, est destinée au dépôt et à la diffusion de documents scientifiques de niveau recherche, publiés ou non, émanant des établissements d'enseignement et de recherche français ou étrangers, des laboratoires publics ou privés. 


\title{
Multi-Object Association with Belief Functions: the Dual Decision Algorithm
}

\author{
J.-P. Lauffenburger, J. Daniel, O. Saif \\ Laboratoire MIPS - EA2332, Université de Haute-Alsace \\ 12 rue des frères Lumière, 68093 Mulhouse Cedex, France \\ (e-mail: jean-philippe.lauffenburger@uha.fr)
}

\begin{abstract}
In intelligent vehicles applications, Multi-Target Tracking (MTT) is usually done considering imperfect sensors' data. As belief functions and particularly the Transferable Belief Model $(T B M)$ help to model these imperfections, it represents an interesting framework for MTT. To carry out target tracking, the system must be able to define at at time $k$ the relations between the detected objects (targets) and the already known ones (tracks). The correlation step is called Multi-Object Association (MOA). This paper tackles the problem of TBM-MOA decision making. The aim is to present a methodology and measures for the management of associations in presence of conflicting sensors' measures. A new algorithm based on a single association matrix gathering all the knowledge of the targets $\Leftrightarrow$ tracks associations is presented. It provides a semantic to avoid the aforementioned difficulties. Simulation results of an obstacle detection application show the advantages of the proposed solution.
\end{abstract}

\section{INTRODUCTION}

A current trend in Intelligent Transportation Systems $(I T S)$ is to provide additional assistance to the user (driver, rider, etc.). As a result, in the automotive domain, numerous Advanced Driver Assistance Systems (ADAS) have been recently commercialized (Adaptive Cruise Control $(A C C)$, Lane Departure Warning System (LDWS), etc.) and now move towards the autonomous vehicle. To perform a safe driving, autonomous vehicles should be aware of their environment and the potential risks which are related to each of its element. They consequently require a correct detection and tracking of the other road users (vehicles, cyclists, etc.) and of static features (traffic signs, driving lines, etc.) to determine the most adapted actions. This environment awareness is usually obtained through the fusion of sensors' information (Daniel and Lauffenburger [2013]), while the tracking is performed by Multi-Target Tracking $(M T T)$. One of the most important step in MTT is the association of the newly detected objects with the already known ones, called Multi-Object Association $(M O A)$. The difficulty is the selection of the most relevant associations.

The origin of MOA lies in the probabilistic domain with the pioneer works of Reid [1979], Blackman and Popoli [1999] and Bar-Shalom [2000]. Usually, MTTs track objects regarding imperfect information (inaccurate, incomplete, conflicting, etc.), i.e. in contexts in which the limits of the probability theory are reached. To cope with these limitations, the Transferable Belief Model (TBM) proposed by Smets and Kennes [1994], can be used. Indeed, the $T B M$ is close to the evidence theory mathematically formalized by Shafer [1976] based on the work of Dempster [1967] concerning the upper and lower probabilities. It is

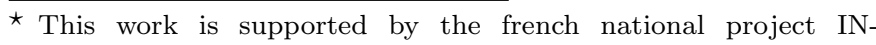
OVE/ANR 2010 BLAN 0308. well suited for imperfect information handling. In most of the recent $M O A$ studies and especially those based on belief functions (Rombaut [1998], Royère et al. [2000], Mourllion et al. [2005]), a two-direction strategy based on independent belief association tables (targets $\Rightarrow$ tracks and vice versa) is adopted. This strategy presented in Fig.1 is particularly useful for the objects appearance and disappearance determination. Moreover, it helps to reveal the contradictions, ambiguities and conflict which may characterize the association problem (Mourllion et al. [2005]).

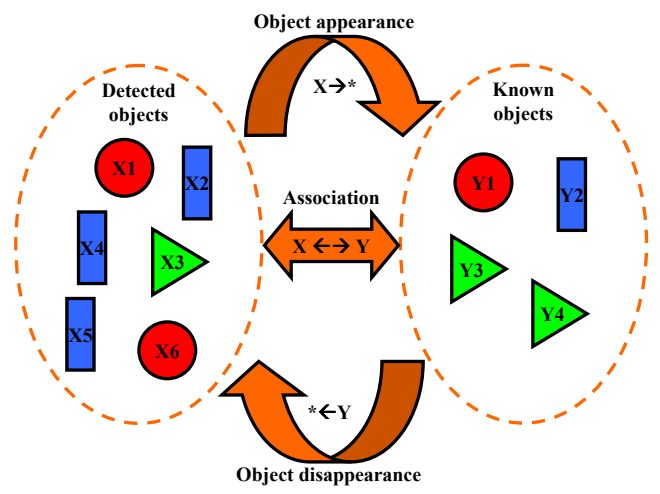

Fig. 1. Association of targets $X_{i}$ with tracks $Y_{j}$.

In Daniel and Lauffenburger [2012], a review of several association strategies in the $T B M$ is presented. It highlights that these strategies are based on the selection of the associations which are globally satisfying the tracking problem. However, it is shown that these solutions could select suspicious local associations and are usually leading to contradictions and ambiguities. To cope with these limitations, two decision algorithms maximizing the local belief of an association have been proposed by the authors. Both algorithms have shown better computation 
time performance and their ability to limit the generation of contradictions. Nevertheless, these problems can still occur in particular cases when evidence conflicts, for instance when a sensor gives a false detection. In addition, the eventual conflict, informing about the difficulty in the objects association represents an interesting piece of information for decision making and should be considered. The present paper focuses on both points by proposing a single association matrix, the dual pignistic matrix which gathers all the association information (targets $\Rightarrow$ tracks and tracks $\Rightarrow$ targets), as well as the conflict related to each of them. Based on the dual pignistic matrix, an association algorithm is described. Simulation comparisons of a real-world case show the advantages of the dual pignistic matrix and especially its ability to avoid contradictions and ambiguities.

The paper is organized as follows: a brief recall of the $T B M$ and its application to $M O A$ is depicted in Section 2. Section 3 presents the principle of the dual pignistic matrix and the proposed association decision algorithm. The latter is evaluated in Section 4. Finally, Section 5 concludes this paper.

\section{TBM MULTI-OBJECT ASSOCIATION}

\subsection{The Transferable Belief Model}

The $T B M$ is a model of uncertain reasoning and decision making based on two levels: the credal level and the pignistic level. In the credal level, belief masses are used to represent the pieces of information whereas, in the pignistic level (or decision level) the belief masses are transformed into probability measures. This section presents the basic elements of the TBM. Additional details can be found in Smets and Kennes [1994].

Consider a problem for which all the discrete solutions (also called hypotheses) $H_{j}, j=1,2, \ldots, K$, with $K$ the number of possible hypotheses, define the frame of discernment $\Theta$ :

$$
\Theta=\left\{H_{1}, H_{2}, \ldots, H_{K}\right\}=\bigcup_{j=1}^{K}\left\{H_{j}\right\}
$$

$\Theta$ is the ignorance, i.e. the union of all hypotheses. Its corresponding referential subset, a power set denoted $2^{\Theta}$ of $2^{K}$ disjunctions of $H_{j}$ is such that:

$$
2^{\Theta}=\left\{\emptyset,\left\{H_{1}\right\}, \ldots,\left\{H_{K}\right\}, \ldots,\left\{H_{1}, H_{2}, H_{3}\right\}, \ldots, \Theta\right\}
$$

$\emptyset$ represents the impossible hypothesis commonly interpreted as the conflict between sources. For practical reasons, the notation $H_{i} \cup H_{j}$ will be preferred to $\left\{H_{i}, H_{j}\right\}$ in this paper. The veracity of a proposition $A$ of $2^{\Theta}$ is characterized by its basic belief mass $(b b m)$ or mass $m$ defined as follows:

$$
\begin{aligned}
m: 2^{\Theta} & \rightarrow[0,1] \\
& A \mapsto m(A), \sum_{A \in 2^{\Theta}} m(A)=1
\end{aligned}
$$

In opposition to the probability theory, the veracity is defined on all disjunctions of $2^{\Theta}$.
The combination gathers the different sources' $b b m$. The most straightforward combination operator is the conjunctive operator $\cap$ (Smets [2007]). By satisfying the associativity as well as commutativity properties, a fusion order definition over the combined sources is not required:

$$
m_{1 \ldots p}^{\cap}(A)=\sum_{A_{1} \cap \ldots \cap A_{p}=A} \prod_{j=1}^{p} m_{j}\left(A_{j}\right)
$$

When disjunctions with an empty intersection are combined $\left(A_{i} \cap A_{j}=\emptyset\right)$, a mass $m_{1 \ldots p}^{\cap}(\emptyset)$ is generated. Usually, the discernment frame is said to be exhaustive and exclusive. Consequently, a mass on the empty set $\emptyset$ is not allowed. Nevertheless, this constraint (known as closed world with $m(\emptyset)=0$ ) is rather difficult to guarantee for real applications. Consequently, two other frameworks have been suggested: the open world (Smets and Kennes [1994]) and the extended open world (Royère et al. [2000]). In Smets' TBM, $\Theta$ is defined as the container of all known hypotheses but not all possible ones $(\Theta$ is exclusive but not exhaustive) so that a mass $m(\emptyset)>0$ holds. In this particular case, $\emptyset$ represents a reject class describing the unknown hypotheses. The conflict related to the nonexhaustivity of the discernment frame can be treated by adding to $\Theta$ an alternative hypothesis $*$ representing all unknown propositions not explicitly defined in $\Theta$ (Royère et al. [2000]). This singleton allows the new discernment frame $\Theta^{*}$ to become exhaustive $\left(\Theta^{*}=\Theta \cup\{*\}\right)$. A non zero value of $m(\emptyset)$ is then only linked to the sources' unreliability or to their discordance.

The selection of the problem solution, i.e. the decision, aims at keeping the most relevant hypothesis $H_{j}$ regarding the combination results. Considering the pignistic level of the $T B M$, the decision can be taken with the Pignistic (or Bet) Probability (BetP) (Smets and Kennes [1994]). The latter is based on a proportional redistribution of the belief of a disjunction over its singletons (cf. (5)). Built as a probabilistic rule, the $B e t P$ is an interesting solution for the $M O A$ context.

$$
\operatorname{BetP}\left(H_{j}\right)=\sum_{\substack{A \in 2^{\Theta} \\ H_{j} \subseteq A}} \frac{m_{1 \ldots p}^{\cap}(A)}{|A|\left(1-m_{1 \ldots p}^{\cap}(\emptyset)\right)}
$$

with $m_{1 \ldots p}^{\cap}(\emptyset)<1$ and $|A|$ the cardinality of the composite $A$. Smets underlines that the pignistic transformation involves a redistribution of the conflict (normalization by $\left.\left(1-m_{1 \ldots p}^{\cap}(\emptyset)\right)\right)$ if a decision must be taken (cf. Smets [2007]). In Section 2.4, this point will be further discussed.

\subsection{Objects Association: Problem Formalization}

Let us consider, as for most of the TBM-MOA (Royère et al. [2000], Mourllion et al. [2005]), specialized sources expressing themselves, for each of them, only over one hypothesis $H_{j}$ of $\Theta$. For a source $j$ expressing itself over the association of a target $X_{i}$ with a track $Y_{j}$, this leads to the following basic belief assignment (bba):

- $m_{j}^{\Theta_{X_{i}}}\left(Y_{j}\right)$ : object $X_{i}$ is associated with object $Y_{j}$, - $m_{j}^{\Theta_{X_{i}}}\left(\bar{Y}_{j}\right)$ : object $X_{i}$ is not associated with object $Y_{j}$, 
- $m_{j}^{\Theta_{X_{i}}}\left(\Theta_{X_{i}}\right)$ : ignorance on the association of $X_{i}$.

$\Theta_{X_{i}}$ and $\Theta_{Y_{j}}$ are the discernment frames referring respectively to the targets $\Rightarrow$ tracks and to the tracks $\Rightarrow$ targets association (cf. (6)). Hence, $\Theta_{X_{i}}$ is composed of the $M$ tracks $Y_{j}$ and the $*$ hypothesis representing a new track. In the same way, $\Theta_{Y_{j}}$ is composed of the $N$ targets $X_{i}$ and the track disappearance hypothesis $*$.

$$
\begin{aligned}
& \Theta_{X_{i}}=\left\{Y_{1}, Y_{2}, \ldots, Y_{M}, *\right\} \\
& \Theta_{Y_{i}}=\left\{X_{1}, X_{2}, \ldots, X_{N}, *\right\}
\end{aligned}
$$

The TBM-MOA looks for the best association regarding the following constraints (Rombaut [1998]):

- a target can only be associated with one track and vice versa,

- appearance and disappearance $(*)$ is possible for multiple targets or tracks.

Here, the focus is placed on the decision making step in the association process. The way the bbas are obtained with respect to the sensor measures is not in the scope of this paper.

\subsection{Combining Belief Masses}

The conjunctive operator $\cap$ described in Section 2.1, is used. As specialized sources are considered, (4) can be formalized for each proposition $H_{j}$ regarding the initial mass distribution $\left(H_{j}, \overline{H_{j}}, \Theta\right)$ as recalled here (Royère et al. [2000], Mourllion et al. [2005]):

$$
\begin{aligned}
& m_{1 \ldots K}^{\cap}\left(H_{j}\right) \quad=m_{j}\left(H_{j}\right) \prod_{\substack{a=1 \\
a \neq j}}^{K} \alpha_{a} \\
& m_{1 \ldots K}^{\cap}\left(H_{j} \cup H_{l}\right)=m_{j}(\Theta) m_{l}(\Theta) \prod_{\substack{a=1 \\
a \neq j \\
a \neq l}}^{K} \gamma_{a}
\end{aligned}
$$

and for union combinations of 2 to $K-1$ hypotheses:

$$
\begin{gathered}
m_{1 \ldots K}^{\cap}\left(H_{j} \cup \ldots \cup H_{l}\right)=m_{j}(\Theta) \ldots m_{l}(\Theta) \prod_{\substack{a=1 \\
a \neq j \\
a \neq i \\
a \neq l}}^{K} \gamma_{a} \\
m_{1 \ldots K}^{\cap}(*) \\
=\prod_{a=1}^{K} \gamma_{a} \\
m_{1 \ldots K}^{\cap}\left(\overline{H_{j}}\right) \quad m_{j}\left(\overline{H_{j}}\right) \prod_{\substack{a=1 \\
a \neq j}}^{K} m_{a}(\Theta) \\
m_{1 \ldots K}^{\cap}(\Theta)=\prod_{a=1}^{K} m_{a}(\Theta) \\
m_{1 \ldots K}^{\cap}(\emptyset)=1-\left[\prod_{a=1}^{K} \alpha_{a}+\sum_{a=1}^{K} m_{a}\left(H_{a}\right) \prod_{\substack{b=1 \\
b \neq a}}^{K} \beta_{b}\right.
\end{gathered}
$$

with $\alpha_{a}=\left(1-m_{a}\left(H_{a}\right)\right), \beta_{b}=\left(1-m_{b}\left(H_{b}\right)\right), \gamma_{a}=$ $\left(m_{a}\left(\overline{H_{a}}\right)\right)$.

\subsection{Pignistic Transformation and Association Matrices}

In TBM-MOA, a situation is observed with two points of view: one is dedicated to the targets-to-tracks $\left(X_{i} \Rightarrow Y_{j}\right)$ association while the second is focused on the tracks-totargets $\left(X_{i} \Leftarrow Y_{j}\right)$ association. In order to face with the association constraints, a pignistic transformation is operated on the combined masses obtained with the generalized rule (7). This leads to two pignistic probabilities matrices shown in Table 1 and 2. Each line defines the probabilities of the associations of $X_{i}$ with $Y_{1}, \ldots, Y_{M}$, * and vice versa. For the sake of clarity, the complete notation $\operatorname{Bet} P_{1 \ldots K}^{\Theta_{X_{i}}}\left(Y_{j}\right)$ (or $\operatorname{Bet} P_{1 \ldots K}^{\Theta_{Y_{j}}}\left(X_{i}\right)$ ) has been simplified to $\operatorname{Bet} P_{X_{i}}\left(Y_{j}\right)$ (or $\left.\operatorname{Bet}_{Y_{j}}\left(X_{i}\right)\right)$.

These matrices are obtained without conflict normalization (contrary to (5)). Indeed, the belief on the empty set $\emptyset$ generated during information combination can be useful for the final decision and is therefore conserved. Section 3.3 describes how this piece of information is used in the pignistic level. Consequently, the pignistic probabilities are given by:

$$
\left\{\begin{array}{l}
\operatorname{BetP}\left(H_{j}\right)=\sum_{\substack{A \in 2^{\Theta} \\
H_{j} \subseteq A}} \frac{m_{1 \ldots K}^{\cap}(A)}{|A|} \\
\operatorname{BetP}(\emptyset)=m_{1 \ldots K}^{\cap}(\emptyset)
\end{array}\right.
$$

The matrices help to detect contradictions and/or ambi-

\begin{tabular}{|c|c|c|c|c|c|}
\hline $\operatorname{Bet} P_{X}$ & $Y_{1}$ & $\ldots$ & $Y_{M}$ & * & $\emptyset$ \\
\hline$X_{1}$ & $\operatorname{Bet}_{X_{1}}\left(Y_{1}\right)$ & $\ldots$ & $\operatorname{Bet}_{X_{1}}\left(Y_{M}\right)$ & $\operatorname{Bet} P_{X_{1}}(*)$ & $\operatorname{Bet}_{X_{X_{1}}}(\emptyset)$ \\
\hline$X_{2}$ & $\operatorname{Bet}_{X_{2}}\left(Y_{1}\right)$ & $\ldots$ & $\operatorname{Bet}_{X_{2}}\left(Y_{M}\right)$ & $\operatorname{Bet} P_{X_{2}}(*)$ & $\operatorname{Bet} P_{X_{2}}(\emptyset)$ \\
\hline : & & : & & & \\
\hline$X_{N}$ & $\operatorname{Bet}_{X_{N}}\left(Y_{1}\right)$ & . & $\operatorname{Bet}_{X_{X}}\left(Y_{M}\right)$ & $\operatorname{Bet}_{X_{N}}(*)$ & $\operatorname{Bet}_{X_{X}}(\emptyset)$ \\
\hline
\end{tabular}
guities in the associations. An ambiguity occurs when, on a given line of a matrix, several probabilities are equal, i.e. several associations are equally possible. On the other hand, a contradiction occurs when the matrices conclude on different associations for the same object. Consequently, ambiguity highlights an intra-matricial association problem whereas contradiction highlights an inter-matricial one.

Table 1. Targets-to-Tracks Pignistic Matrix

Table 2. Tracks-to-Targets Pignistic Matrix

\begin{tabular}{|c|c|c|c|c|c|}
\hline $\operatorname{Bet} P_{Y_{j}}$ & $X_{1}$ & $\ldots$ & $X_{N}$ & $*$ & $\emptyset$ \\
\hline$Y_{1}$ & $\operatorname{Bet} P_{Y_{1}}\left(X_{1}\right)$ & $\ldots$ & $\operatorname{Bet} P_{Y_{1}}\left(X_{N}\right)$ & $\operatorname{Bet} P_{Y_{1}}(*)$ & $\operatorname{Bet} P_{Y_{1}}(\emptyset)$ \\
\hline$Y_{2}$ & $\operatorname{Bet} P_{Y_{2}}\left(X_{1}\right)$ & $\ldots$ & $\operatorname{Bet} P_{Y_{2}}\left(X_{N}\right)$ & $\operatorname{Bet} P_{Y_{2}}(*)$ & $\operatorname{Bet} P_{Y_{2}}(\emptyset)$ \\
\hline$\vdots$ & $\vdots$ & $\vdots$ & $\vdots$ & $\vdots$ & $\vdots$ \\
\hline$Y_{M}$ & $\operatorname{Bet} P_{Y_{M}}\left(X_{1}\right)$ & $\ldots$ & $\operatorname{Bet} P_{Y_{M}}\left(X_{N}\right)$ & $\operatorname{Bet} P_{Y_{M}}(*)$ & $\operatorname{Bet} P_{Y_{M}}(\emptyset)$ \\
\hline
\end{tabular}

\section{DUAL DECISION ALGORITHM}

\subsection{MOA Decision Making}

In Daniel and Lauffenburger [2012], the authors have compared different decision algorithms focusing on the 
previously introduced pignistic matrices. Three of them are based on maximizing the global belief criteria. The results showed the limitations of such approaches, leading the authors to the definition of two local decision algorithms. The first one - the Local Pignistic Probability $(L P P)$ - is based on the line-wise selection of the $N / M$ (depending on the association direction) pignistic probability maximum. The $L P P$ has been shown to be less time consuming compared to algorithms from the literature since no extra computation is required for the decision making. The second algorithm is the Gradient Classified Pignistic Probability $(G C P P)$ which behaves similarly to the $L P P$ but, before the associations selection, a preliminary classification of the pignistic matrix is done with respect to the belief gradient in a line. This approach is interesting as the pignistic gradient reveals the uncertainty related to the considered associations. The advantage of these local algorithms mainly lies in their ability to limit the ambiguities and contradictions and the selection of non suspicious local associations. However, by analyzing the scene from two different points of view, all these solutions can face with association difficulties. Moreover, it is worth noting that the conflict is not considered in these decision solutions.

\subsection{Two-direction Association Limitations}

The two-direction association allows to treat all the targets and tracks independently, so that each of them is characterized by association, appearance/disappearance and conflict masses. This approach also reveals the eventual ambiguities and contradictions which characterize the association problem. The question raised is then: how to manage these contradictions and ambiguities? In Daniel and Lauffenburger [2012], the authors have shown that the selection of the decision algorithm can limit these effects by retaining the appropriate local associations in both pignistic matrices. Nevertheless, in particular cases, especially in conflicting situations, another solution has to be found. This paper describes how the fusion of both pignistic matrices into a generic one, called dual pignistic matrix storing all the association and conflict information, can help.

\subsection{Dual Pignistic and Dual Conflict Matrices}

Both pignistic matrices (Table 1 and Table 2) give information about the potential associations either for the targets or the the tracks. The advantage in combining these matrices is to solve the contradictions and ambiguities by providing a single information matrix storing all the association data. This approach, described in Mourllion et al. [2005] with the global pignistic matrix, was based on the computation of the mean pignistic probability from the $\operatorname{Bet} P_{X_{i}}$ and $\operatorname{Bet}_{Y_{Y_{j}}}$ matrices as presented in (9). Considered as a cost matrix, the global pignistic matrix is used in a Hungarian algorithm for decision making. The limitations of this approach are the loss of the conflict information due to the normalization of the combined masses, the use of a global optimization resolution technique and finally the averaging of the pignistic probabilities.

$$
M(i, j)=\frac{\operatorname{Bet}_{X_{i}}\left(Y_{j}\right)+\operatorname{Bet} P_{Y_{j}}\left(X_{i}\right)}{2}
$$

In the present paper, the authors propose to cope with these limitations through the definition of a general matrix, called dual pignistic matrix, taking account of the conflict. The first difference lies in the calculation of the dual association values. Contrary to Mourllion et al. [2005], the dual association matrix is computed through the Hadamard product of the local pignistic probabilities $\operatorname{Bet}_{Y_{j}}($.$) and \operatorname{Bet} P_{X_{i}}($.$) as presented in (10). In a$ first step, the pignistic matrix describing the targets-totracks association $B e t P_{X_{i}}($.$) is transposed to verify matrix$ dimension properties. The operator $\circledast$ is introduced to represent the element-wise Hadamard product. The resulting matrix is composed of the pignistic probabilities $M_{Y X}^{D u a l}(i, j)$. It will be further related to dual pignistic probabilities.

$$
M_{Y X}^{\text {Dual }}=\left(\operatorname{Bet} P_{X_{i}}(.)\right)^{T} \circledast \operatorname{Bet} P_{Y_{j}}(.)
$$

Multiplying the two pignistic matrices $\operatorname{Bet} P_{X_{i}}($.$) and$ $B$ et $P_{Y_{j}}$ (.) in this way induces a significant penalty on the pignistic probabilities. This is the key to overcome association contradictions and ambiguities. For instance, if an association is certain, i.e. characterized by high pignistic probabilities in both matrices, the dual pignistic probability will be high. In case of a contradiction defined by a high pignistic probability in one matrix and low in the other, the Hadamard product will lead to the reduction of the belief in this association. Similarly, when an ambiguity occurs (when two pignistic probabilities are equal in the same line either in $\operatorname{Bet} P_{X_{i}}($.$\left.) or in \operatorname{Bet}_{Y_{j}}().\right)$, the dual pignistic matrix will permit to select the best association. Being more conservative, this model presents a better discrimination power than the global pignistic matrix formalized in (9). Indeed, associations characterized by low $B$ et $P$ in the local matrices will lead to an even lower value in the dual pignistic matrix, while confident associations would still be characterized by a high dual value.

This paper also proposes to consider the conflict in the decision matrix. Therefore, it is integrated as an additional line and column in the dual pignistic matrix. This measure characterizes the conflict level between one target/track and all the tracks/targets. The pignistic values of the conflict $\operatorname{Bet}_{Y_{Y_{j}}}(\emptyset)$ and $\operatorname{Bet} P_{X_{i}}(\emptyset)$ are first combined in order to define the dual conflict matrix $M_{Y X}^{\text {Conf }}$ such that:

$$
M_{Y X}^{C o n f}=\emptyset_{Y X} \cdot\left(\emptyset_{X Y}\right)^{T}
$$

with $\emptyset_{Y X}=\operatorname{Bet} P_{Y_{j}}(\emptyset)$ and $\emptyset_{X Y}=\operatorname{Bet} P_{X_{i}}(\emptyset)$.

$M_{Y X}^{C o n f}$ reveals important information about conflictrelated associations. In fact, the cells of this matrix represent the dual conflict probability over all individual possible associations (one track $\Leftrightarrow$ one target). By analyzing this matrix, the conflicting associations can be easily known. The dual conflict linked to each target and track is then respectively obtained through the matrix row and column sum (cf. (12)). The dual conflict vector $V_{Y X}^{\text {Conf }}$ related to the $Y_{j} \Rightarrow X_{i}$ associations is the result of the sum of the dual conflict matrix columns. Similarly, the dual vector of the conflict for the $X_{i} \Rightarrow Y_{j}$ associations $V_{X Y}^{C o n f}$ is the result of the sum of the columns of the transposed matrix. The elements of these vectors give an accurate 
information respectively on the targets/tracks affected by conflict and the dual pignistic probability of this conflict.

$$
\begin{aligned}
& V_{Y X}^{\text {Conf }}(.)=\sum_{i=1}^{M} M_{Y X}^{\text {Conf }}(i, .) \\
& V_{X Y}^{\text {Conf }}(.)=\sum_{i=1}^{N}\left(M_{Y X}^{\text {Conf }}\right)^{T}(i, .)
\end{aligned}
$$

Both vectors complete the dual pignistic matrix as presented in Table 3. Note that a zero value is added to the the matrix so that it becomes square.

Table 3. Dual Pignistic Matrix

\begin{tabular}{|c|c|c|c|c|}
\hline$M_{Y X}^{\text {Dual }}$ & $X_{1}$ & $\ldots$ & $X_{N}$ & $V_{Y X}^{\text {Conf }}$ \\
\hline$Y_{1}$ & $M_{Y X}^{\text {Dual }}(1,1)$ & $\ldots$ & $M_{Y X}^{\text {Dual }}(1, N)$ & $V_{Y X}^{\text {Conf }}(1)$ \\
\hline$Y_{2}$ & $M_{Y X}^{\text {Dual }}(2,1)$ & $\ldots$ & $M_{Y X}^{\text {Dual }}(2, N)$ & $V_{Y X}^{\text {Conf }}(2)$ \\
\hline$\vdots$ & $\vdots$ & $\ddots$ & $\vdots$ & $\vdots$ \\
\hline$Y_{M}$ & $M_{Y X}^{\text {Dual }}(M, 1)$ & $\ldots$ & $M_{Y X}^{\text {Dual }}(M, N)$ & $V_{Y X}^{\text {Conf }}(M)$ \\
\hline$V_{X Y}^{\text {Conf }}$ & $V_{X Y}^{\text {Conf }}(1)$ & $\ldots$ & $V_{X Y}^{\text {Conf }}(N)$ & 0 \\
\hline
\end{tabular}

\subsection{Dual Decision Algorithm}

Based on the dual pignistic matrix which gathers all the association information, a new decision algorithm is proposed in this paper (cf. Algorithm 1). The conflictbased Dual Decision algorithm consists in the linewise selection of the dual pignistic probability maximum of $M_{Y X}^{\text {Dual }}(j,$.$) (each of them being related to one track Y_{j}$ ). These values are then compared to the dual conflict to determine if the situation allows an association or not. Indeed, a high conflict reveals an association problem which can be for instance due to a source false information. If the maximum value is higher than the conflict, then it is compared to a Threshold to determine if the object is associated or if it disappears. Finally, the targets $X_{i}$ which have not been associated are considered to appear or are not associated, depending on the dual conflict level.

\section{APPLICATION TO VEHICLE DETECTION AND TRACKING}

\subsection{Test Scenario}

Let us consider the example from Mourllion et al. [2005] presented in Fig.2. Four known vehicles $\left(Y_{j}\right)$ are considered but two of them are outside of the detection range $\left(Y_{3}\right.$ and $\left.Y_{4}\right)$, thus are subject to disappearance. It can be noted that among the three detections, one corresponds to a false alarm due to the closeness of $Y_{1}$ and $Y_{2}$. For this example the sources are expressing themselves as shown in Table 4.

These bbas lead to ambiguities since $Y_{1}$ can be associated either with $X_{1}$ or $X_{2}$, while $Y_{2}$ can be associated either with $X_{2}$ or $X_{3}$. On the other side, $X_{2}$ can be associated either to $Y_{1}$ or $Y_{2}$. (13) sums up the potential associations induced by this example.

$$
\begin{aligned}
& \begin{array}{lll|llll}
X_{1} & X_{2} & X_{3} & Y_{1} & Y_{2} & Y_{3} & Y_{4}
\end{array}
\end{aligned}
$$

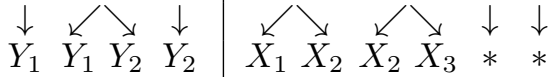

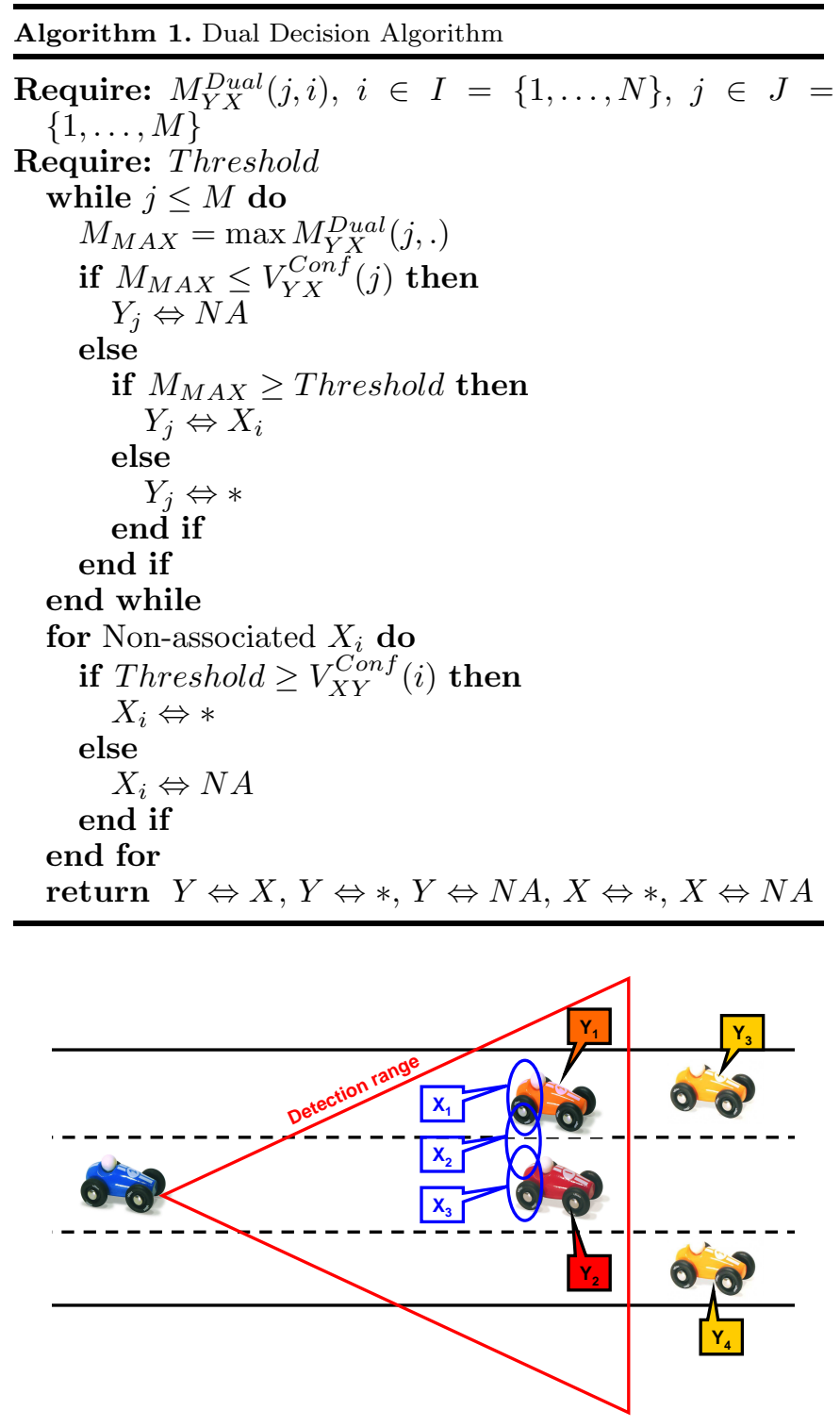

Fig. 2. Case study: $X_{2}$ is a sensor false detection. $Y_{3}$ and $Y_{4}$ are out of the sensor's range.

\subsection{Association Results}

For this example, the discernment frames are:

$$
\begin{gathered}
\Theta_{X_{i}}=\left\{Y_{1}, Y_{2}, Y_{3}, Y_{4}, *\right\} \\
\Theta_{Y_{j}}=\left\{X_{1}, X_{2}, X_{3}, *\right\}
\end{gathered}
$$

Table 4. Initial belief masses

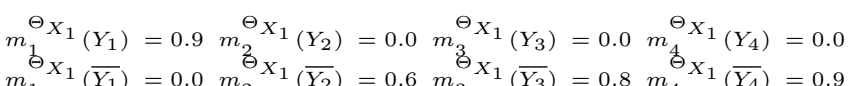

$$
\begin{aligned}
& \begin{array}{ll}
m_{1}{ }_{\Theta_{1}}\left(\overline{Y_{1}}\right)=0.0 & m_{2}{ }_{\Theta_{1}}\left(\overline{Y_{2}}\right)=0.6 m_{3}\left(\overline{Y_{3}}\right)=0.8 m_{4} m_{X_{1}}\left(\overline{Y_{4}}\right)=0.9 \\
m_{1}{ }_{1}(\Theta)=0.1 & m_{2}{ }^{1}(\Theta)=0.4 m_{3}{ }_{X_{1}}(\Theta)=0.2 m_{4}{ }_{X_{1}}(\Theta)=0.1
\end{array} \\
& m^{\Theta_{X_{2}}}\left(Y_{1}\right)=0.6 m_{2}^{\Theta_{X_{2}}}\left(Y_{2}\right)=0.6 m_{3}^{\Theta_{X_{2}}}\left(Y_{3}\right)=0.0 m_{4}^{\Theta_{X_{2}}}\left(Y_{4}\right)=0.0 \\
& \left.\left.m_{X_{2}}\left(\overline{Y_{1}}\right)=0.0 m_{X_{2}}^{\Theta_{\left(Y_{2}\right.}}\right)=0.0 m_{3}^{\Theta_{X}}\left(\overline{Y_{3}}\right)=0.8 m_{X_{2}}^{\Theta_{Y_{4}}}\right)=0.9 \\
& m_{1}^{\Theta_{X}}(\Theta)=0.4 m_{2}{ }^{\Theta_{2}}(\Theta)=0.4 m_{3}^{\Theta_{X}}(\Theta)=0.2 m_{4}^{\Theta_{X}}(\Theta)=0.1
\end{aligned}
$$

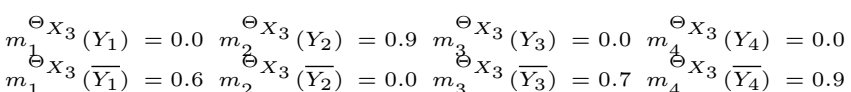

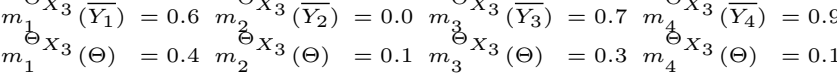


The combination defining the association matrix $\operatorname{Bet} P_{X_{i}}($. is performed line-wise whereas $\operatorname{Bet} P_{Y_{j}}($.$) is obtained by a$ column-wise combination using the generalized rules (7) and the pignistic transformation (8). Tables 5 and 6 give respectively the $X_{i} \Rightarrow Y_{j}$ and $Y_{j} \Rightarrow X_{i}$ probabilities.

Table 5. Targets-to-Tracks Probabilities

\begin{tabular}{|c|c|c|c|c|c|c|}
\hline Bet $P_{X_{i}}()$. & $Y_{1}$ & $Y_{2}$ & $Y_{3}$ & $Y_{4}$ & $*$ & $\emptyset$ \\
\hline$X_{1}$ & 0.94 & 0.01 & 0.01 & 0.00 & 0.04 & 0.00 \\
\hline$X_{2}$ & 0.29 & 0.29 & 0.01 & 0.00 & 0.05 & 0.36 \\
\hline$X_{3}$ & 0.01 & 0.94 & 0.01 & 0.00 & 0.04 & 0.00 \\
\hline
\end{tabular}

Table 6. Tracks-to-Targets Probabilities

\begin{tabular}{|c|c|c|c|c|c|}
\hline $\operatorname{Bet}_{Y_{j}}()$. & $X_{1}$ & $X_{2}$ & $X_{3}$ & $*$ & $\emptyset$ \\
\hline$Y_{1}$ & 0.37 & 0.07 & 0.00 & 0.01 & 0.54 \\
\hline$Y_{2}$ & 0.00 & 0.07 & 0.37 & 0.01 & 0.54 \\
\hline$Y_{3}$ & 0.08 & 0.08 & 0.13 & 0.70 & 0.00 \\
\hline$Y_{4}$ & 0.05 & 0.05 & 0.05 & 0.86 & 0.00 \\
\hline
\end{tabular}

These matrices confirm the association assumptions described in (13) revealing the difficulty, except for $Y_{3}$ and $Y_{4}$ which should disappear $\left(\operatorname{Bet} P_{Y_{3}}(*)=0.70\right.$ and $\left.\operatorname{Bet} P_{Y_{4}}(*)=0.86\right)$. The association of $X_{2}$ is particularly interesting. Indeed, $X_{2}$ is subject to conflict $\left(\operatorname{Bet} P_{X_{2}}(\emptyset)=\right.$ 0.36 ) and leads to an ambiguity since $X_{2}$ is associated to $Y_{1}$ and to $Y_{2}\left(\operatorname{Bet} P_{X_{2}}\left(Y_{1}\right)=\operatorname{Bet} P_{X_{2}}\left(Y_{2}\right)=0.29\right)$. In addition, Table 6 shows the difficulty of associating $Y_{1}$ and $Y_{2}$ as they are characterized by high and equivalent conflict (0.54). Both BetP matrices are then used to calculate the dual pignistic matrix using equations (10), (11) and (12) leading to Table 7 . Its conservative aspect is highlighted by the low probabilities, except for the $Y_{1} \Leftrightarrow X_{1}, Y_{2} \Leftrightarrow X_{3}$ $\left(M_{Y X}^{\text {Dual }}(1,1)=M_{Y X}^{\text {Dual }}(2,3)=0.35\right)$. In addition, a high value of dual conflict can be noticed for the association of $X_{2}\left(V_{X Y}^{\text {Conf }}(2)=0.39\right)$. This conflicting example is consequently highlighting the good properties of the dual pignistic matrix.

Table 7. Dual Pignistic Matrix

\begin{tabular}{|c|c|c|c|c|}
\hline$M_{Y X}^{\text {Dual }}$ & $X_{1}$ & $X_{2}$ & $X_{3}$ & $V_{Y X}^{\text {Conf }}$ \\
\hline$Y_{1}$ & 0.35 & 0.02 & 0.00 & 0.19 \\
\hline$Y_{2}$ & 0.00 & 0.02 & 0.35 & 0.19 \\
\hline$Y_{3}$ & 0.00 & 0.00 & 0.01 & 0.00 \\
\hline$Y_{4}$ & 0.00 & 0.00 & 0.00 & 0.00 \\
\hline$V_{X Y}^{\text {Conf }}$ & 0.00 & 0.39 & 0.00 & 0.00 \\
\hline
\end{tabular}

The threshold value required by the algorithm has been fixed to 0.1 due to the conservative behavior of the dual pignistic matrix. The association relation depicted in (15) presents the results of the dual decision algorithm. These are intuitive and concordant to the predefined ones. Indeed, $Y_{3}$ and $Y_{4}$ are disappearing, while $Y_{1}$ and $Y_{2}$ are respectively associated with $X_{1}$ and $X_{3}$. Then, $X_{2}$, characterized by an ambiguity in the pignistic matrices, has not been associated at all and is discarded, due to the high dual conflict $\left(V_{X Y}^{\operatorname{Conf}}(2)=0.39\right)$. This makes sense since a high conflict value leads to a difficult decision making, which is here coming from a sensor false detection.

$$
\begin{array}{cccc|c}
Y_{1} & Y_{2} & Y_{3} & Y_{4} & X_{2} \\
\downarrow & \downarrow & \downarrow & \downarrow & \downarrow \\
X_{1} & X_{3} & * & * & N A
\end{array}
$$

\section{CONCLUSION}

This paper tackled the problem of Multi-Object Association $(M O A)$ performed within the Transferable Belief Model $(T B M)$. A new decision algorithm avoiding contradictions and ambiguities usually present in traditional approaches is proposed. To cope with these problems, the present paper described the determination of a single decision measure, the dual pignistic matrix, storing the information related to the bi-directional associations. A conflict measure related to each target or track is also presented. To deal with the information stored in this single matrix, a decision algorithm has been developed for the selection of the most relevant associations. This algorithm selects the associations with a dual pignistic probability higher than a threshold, taking account of the conflict. Simulations, reproducing a real driving situation, show the ability of the proposed method to avoid contradictions and ambiguities.

The dual pignistic matrix could be enhanced to also directly integrate the information related to objects appearance and/or disappearance available after source combination. In this paper, the one-to-one association constraints lead to a mono-hypothesis tracking algorithm. By letting the algorithm being undecided, i.e. some objects could not be associated at all, multi-hypothesis tracking will be possible in future works.

\section{REFERENCES}

Y. Bar-Shalom. Multitarget-multisensor tracking: Applications and Advances, volume III. Artech House, 2000.

S. Blackman and R. Popoli. Design and Analysis of Modern Tracking Systems. Artech House, 1999.

J. Daniel and J.-P. Lauffenburger. Multi-object association decision algorithms with belief functions. In Fusion, Singapore, 2012.

J. Daniel and J.-P. Lauffenburger. Fusing navigation and vision information with the transferable belief model: Application to an intelligent speed limit assistant. Informat. Fusion, 2013. doi: http://dx.doi.org/10.1016/j.inffus.2013.05.013.

A.P. Dempster. Upper and lower probabilities induced by a multivalued mapping. Annals of Mathematical Statistics, 38:325-339, 1967.

B. Mourllion, D. Gruyer, C. Royère, and S. Théroude. Multi-hypotheses tracking algorithm based on the belief theory. In Fusion, Philadelphia, PA, USA, 2005.

D.B. Reid. An algorithm for tracking multiple targets. IEEE Trans. on Automatic Control, 24:843-854, 1979.

M. Rombaut. Decision in multi-obstacle matching process using Dempster-Shafer's theory. In AVCS, Amiens, France, 1998.

C. Royère, D. Gruyer, and V. Cherfaoui. Data association with belief theory. In Fusion, Paris, France, 2000.

G. Shafer. A mathematical theory of evidence. Princeton University Press, 1976.

P. Smets. Analyzing the combination of conflicting belief functions. Information Fusion, 8:387-412, 2007.

P. Smets and R. Kennes. The transferable belief model. Artificial Intelligence, 66:191-234, 1994. 